Aus der chirurgischen Abteilung des Alt-Katharinenkrankenhauses in Moskau.

\title{
Über einen Fall von Unterbindung der Art. ano- nyma infolge eines arteriovenösen Aneurysma der Art. carotis communis.
}

\author{
Von P. Herzen.
}

Die Unterbindung des Truncus anonymus ist eine selten indizierte und auch selten ausgeführte Operation. In seiner ausgezeichneten Monographie hat Prof. Wwedensky 3 I Fälle solcher Unterbindungen der Anonyma zusammengestellt; leider sind nicht alle genügend dokumentiert worden. Sh e e $\mathrm{n}$ unterband im Jahr I905 die Anonyma - sein Fall war der 37. in der Literatur. Im Jahre I 908 führt $\mathrm{B}$ u r n s eine Statistik von $5 \mathrm{I}$ Fällen an und beschreibt einen neuen Fall einer gut gelungenen Unterbindung der Anonyma. Freilich berichtet er über II Fälle, von denen ein Teil nicht zu Ende geführt wurde, bei den anderen konnte man nicht genau feststellen ob wirklich der uns interessierende Truncus unterbunden worden ist. Somit bleiben inklusiv mit dem Falle B u $\mathrm{rns}$ nur noch 4I. Diese von uns vorgenommene Operation ist eine sehr gefährliche. Prof. Wwe $\mathrm{d}$ ens ky fand nur 8 gutgelungene Operationen. Von Burns 5 I Fälle kamen nur I2 oder richtiger gesagt von $4 \mathrm{I}$ nur Io zur Genesung. Somit bietet ein jeder neue Fall von Unterbindung der Art. anonyma ein großes Interesse dar, hauptsächlich wenn die Operation gut gelingt, da sie ein neues Licht auf die dunklen und gefährlichen Seiten der nicht in allen großen Handbüchern der Chirurgie beschriebenen Operationen wirft.

M e i n F a ll. Sergei T., 30 Jahre alt, Schutzmann. Wurde den I5. IX, I908 in der Chirurgischen Abteilung des Altkatharinen-Hospitals zu Moskau aufgenommen. Den I3. V. Igo8 bekam der Kranke 3 Schußwunden aus dem Browning auf einer Entfernung von 3 Schritte. Nach 
der ersten Schußwunde in den Hals verteidigte er sich noch, aber nach zwei folgenden Wunden in den rechten Arm und das rechte Bein brach er zusammen. In einer Droschke wurde der Verwundete ins Krankenhaus gebracht. Unterwegs stürzte er aus dem Wagen und brach sich das rechte Schlüsselbein. Bei weiterem Ausfragen erfuhren wir, daß die Schußwunde am Halse heftig blutete. Im Hospital zu Tambow hatte der Kranke bewußtlos 3 Tage gelegen. Die Wunden heilten unter dem Verbande und nach 7o Tagen verließ der Kranke das Hospital mit einer Paralyse der rechten Hand und heftigen Schmerzen in der rechten Hälfte des Kopfes, des Halses und der rechten Hand. Nach der Verwundung bemerkte der Kranke einen sausenden diffusen Tumor in der Suprasternalgruppe. Allmählich bemerkte man ein langsam wachsendes Aneurysma, daß mit einer Schwellung der rechten Wange und des rechten Armes einherging. Der Kranke beklagte sich über das laute Sausen, welches ihṇ im Schlafe störte. Zu gleicher Zeit empfand der Kranke, daß ihm der Halskragen seines Rockes zu eng wurde.

St. pr. Der Kranke ist gut genährt und gut gebaut.

Die inneren Organe sind normal.

Beträchtliche Atrophie des Mm. deltoideus und der Muskulatur des rechten Oberames. Odem und Cyanose des rechten Vorderarmes und der rechten Hand. Folgende Narben infolge der Schußwunden: I. Hinten, auf der Grenze zwischen mittlerem und unterem Drittel des rechten Armes und auf der lateralen Seite des rechten Vorderarmes. 2. Auf dem rechten Unterschenkel unter der Spina ant. sup. ossis ilei. Keine Ausgangsnarbe vorhanden. 3. Lateralwärts der Trachea in der Höhe des ersten Trachealringes befindet sich die Narbe der Eingangswunde; eine Ausgangswunde fehlt. Die Kugel befindet sich unter dem inneren Winkel des rechten Schulterblattes. Im Bereiche der Eingangswunde, d. h. zwischen dem inneren Rande des inneren Drittels des M. strno. cl. mastoid. und auch ein wenig unter ihm einerseits und der Trachea unter der Schilddrüse anderseits befindet sich ein kugelförmiger Tumor, dessen untere Grenze hinter der Articulatio sterno clavicularis verschwindet. Der wallnußgroße Tumor ist hart, pulsiert. Beim Pulsieren erweitert er sich expansiv und ist mit der Trachea und den Muskeln verbunden. Beim Anlegen sowohl der bloßen Hand als auch des Stethoskops hört man ein mit dem Pulse synchronisches Gurren. Weiter fühlt man in seiner Mitte einen irregulär verwachsenen Bruch des rechten Schlüsselbeines. Die Bewegungen im Ellenbogen und Handgelenke des rechten Armes sind stark begrenzt. Der Kranke kann den Arm im Schultergelenke dank der Kontraktion des M. cucullaris ein wenig heben. Die aktiven Bewegungen im Schultergelenke fehlen vollständig, die passiven aber sind vorhanden. Der Kranke ist imstande kleine Flexionsbewegungen in dem Handgelenke hervorzurufen, während andere wie passive so auch aktive ausgeschlossen sind. Die Fingergelenke sind fast ankylotisch, die Finger selbst, gleich 
kleinen Stöcken, kann der Kranke nur mit Mühe bewegen. Atıf dem Vorderarme und der Haut im Bereiche der Inervation des N. radialis ist die Empfindlichkeit aufgehoben. Beim ersten Blicke auf den kranken tritt in auffälliger Weise eine Cyanose und Odem des Ohres, der Wange und des oberen Halsteiles hervor. Das auf dem Aneurysma hörbare Gurren pflanzt sich auf die fortlaufende Venen subcl. dext. und axill. dextr. fort; auch ist es auf der ödemen Wange zu hören. Die rechte Art. temporalis pulsiert nicht, in der linken fühlt man den Puls aber ganz deutlich. Der Radialpuls wie der rechten so auch der linken Hand ist ganz deutlich zu fühlen, er ist synchronisch. Die Hautvenen der rechten Seite des Halses sind bedeutend dilatiert. Der Umfang des Halses ist $42 \mathrm{~cm}$. breit

Den I7. IX. hat der Nervenarzt zu unserem Befunde noch folgendes hinzugefügt: Bedeutende muskuläre Atrophie des rechten Armes, hauptsächlich in der Schulter. In der Hand eine myogene extensorische Kontraktur. Sehr starker Schmerz beim Palpieren der Nerven. Der Sehnenreflex des biceps und des triceps fehlt ganz. Di a g nose : Neuritis plexus brachialis dextra traumatica. Dem Anscheine nach ist der Plexus brachialis fast gänzlich zerquetscht und eingeklemmt.

4. X. Operation unter Äther-Chloroformnarkose nach B i $11 \mathrm{rot}$ h. Ein Schnitt längs der Medianlinie des Halses bis zur Mitte des Sternum, dazu ein Horizontalschnitt längs der Clavicula bis zu ihrer Mitte. Alle Venen des Halses bluten stark mit einer pulsierenden Welle; sie werden alle mit Seite unterbunden. Beide winkelförmige Hautlappen werden nach oben und nach unten zurückgeklappt. Subperiostal wird das innere Drittel der Clavicula reseziert und der M. st. cloid. mast. lateralwärts nach außen zurückgeschlagen. Die Fascia über dem Jugulum wird quer durchschnitten, das Periost der hinteren Fläche des manubrii sterni wird mit Hilfe des Raspators und dem Finger abgelöst. und ein Teil dieses Knochens mit der Luerschen Zange reseziert. Jetzt wird die Operationswunde besichtigt. Unter dem clavicularen Ende des M. st. cl. mast. zwischen ihm und der Trachea pulsiert das Aneurysma, ein wallnußgroßer Sack, der mit einem fibrösen Bindegewebe mit dem M. st. cl. mast. und der Trachea stark verwachsen ist. Das ganze untere Drittel der Fossa jugularis ist diffus von fibrösen Bindegewebe eingenommen, in dem sich die Muskeln Ster. hyoid. und St. thyreoideus medianwärts und Omohyoideus oben befinden. Die zwei erstgenannten Muskeln werden nach dem Durchschneiden ihrer Sternalenden medianwärts und nach oben gezogen.

Nach einem Horizontalschnitte der Fascia endothoracico versuchte man den Ausgangspunkt der Art. carotis comm. freizulegen; das gelang aber nicht, da das Fettgewebe des Paratrachealraumes stark verwachsen war. Dann ging man nach dem Aufschneiden des hinteren Periost mahubrii sterni mit stumpfen Instrumenten und dem Finger in die Tiefe des Mediastini antici. Das Auffinden der Art. anonyma ist sehr 
schwer, da die Arterie wie auch die Vene anonyma pulsieren und sausen, außerdem sind sie noch von einem recht festen Bindegewebe eingeschlossen. Endlich gelang es, sich der Medianlinie und nahe der Art. carotis communis haltend, die vordere Fläche der Art. anonyma zu finden. Die rechte wie auch die linke Vene anonyma werden nicht freigelegt sondern mit dem Zellgewebe weitergeschoben. Die vordere und die lateralen Seiten der Arterie werden freigelegt, aber das Freilegen der hinteren Seite wiedersteht sogar den Instrumenten. Man ist genötigt, eine recht dicke Schicht des hinteren Zellgewebes mit der Deschampschen Nadel durchzustechen. Mit einem durchgeführten Seidenfaden wird die Art. unterbunden.

Beim Aufsuchen der Art. anonyma wird die Pleura infolge starker Spannung der Adhäsionen aufgerissen, diese Stelle aber kann man nicht zu Gesicht bekommen und das ein- und ausströmen der Luft wird mit dem Tamponieren des Fingers teilweise verhindert. Nach der Unterbindung der Art. anonyma hört das Gurren in dem Aneurysma auf, zu gleicher Zeit versagt auch der rechte Radialpuls. Jetzt wird der M. omo-hyoideus durchgeschnitten und die großen Gefäße des Halses oberhalb des Aneurysma freigelegt. Die Vene jugularis communis, sehr stark bis zum Umfange eines Dünndarmes gedehnt, wird zwischen zwei Ligaturen durchschnitten. Dessen ungeachtet füllt sich das zentrale Ende der Vene bis zum früheren Umfange mit Blut. Die Art. carotis communis wird gleichfalls zwischen zwei Ligaturen durchgeschnitten aber das Aneurysma füllt sich dennoch mit zurückfließendem Blute aus der Art. subclavia und der Vene. Dann wird die Anonyma an der Stelle ihrer Teilung in die Art. subclavia und der Art. carotis freigelegt und die letztere dicht bei ihrem Ausgange unterbunden und durchschnitten. Jetzt geht man zur Exstirpation des Aneurysma über. Von oben geht das Exstirpieren recht leicht, es gelingt den N. vagus von dem Sacke freizulegen; im Gegenteil das Abtrennen (die Isolierung) des Sackes von der Trachea gibt starke Blutung und wird einstweilen unterlassen. Um zweifellose Blutstillung zu erzeugen geht man von der unteren Seite des Aneurysma auf den Bulbus venosus; es gelingt aber nicht ihn $\mathrm{zu}$ isolieren, da er mit der V. subclavia und $V$. jugularis von einem dichten fibrösen Bindegewebe umhüllt ist, welches vom Aneurysma längs dem Kugeltraktus auf dem Bulbus sich erstreckt. Man ist genötigt mit nicht geringer Gewalt en masse eine seidene Ligatur anzulegen, die den oberen Teil des Bulbus bei der Mündung der V. jugularis in die V. subclavia umschlingt. Erst jetzt gelingt es nicht ohne Mühe, aber ohne Blutverlust die Exstirpation des Aneurysma zu beenden.

Die kleinen Gefäße Vena und Art. thyreoidea ima werden unterbunden. Auf das Zellgewebe auf Mm. sterno-hyoid. und sterno thyreoid, die über die Stelle, wo die Luft aus der Pleura herausging, werden Nähte angelegt. Der Muskel St. cl. mast. wird mit dem Periost der Clavicula 
und dem Manubrium sternii mit Naht verbunden. Nähte auf die Haut. 2 Tampons. Trockener Verband. Die Operation hat der Kranke gut überstanden. Der Puls war nicht wesentlich verändert. Abends Temp. 37,3.

Am 5. X. 37-38,6. Klagen über Husten. Puls beschleunigt aber voll. Das Allgemeinbefinden befriedigend. Das Atmen ruft Schmerzen hervor. Pleuritische Reibegeräusche. Die Tampons werden entfernt. Es floß recht viel blutiggefärbte Flüssigkeit heraus die den Verband während der Nacht durchnäßt hat. Der Puls ist auf der rechten Hand nicht fühlbar. Rp.: Infus Ipcac.e o,6: 18o Liq. ammon. anisat. 9,0.

6. $37,8-38,0$.

7. 37,6--37,6. F a s t kein Ausscheiden aus der Wunde.

8. 37,3-38,4. Pleuritische Reibegeräusche noch zu hören. Die Finger der rechten Hand sind ein wenig cyanotisch.

9. 37,6-38,3. Hinten rechts unter der Scapula eine Dämpfung und Brochialatmen. Rings der Naht eine starke Reizung. Puls ist gut.

Io. 37,4-38,o. Puls ist gut. Die Nähte werden entfernt. Prima intentio. Die Reizung an der Naht ist geringer. In der rechten Lunge hört man hinten Bronchialatmen und Rasselgeräusche. Rp.: Camph. o,I, - Chinin. muriat. $0,2 \mathrm{~N} 4$.

II. 37,4-38,4. Kein Stuhlgang. Ol. Ricin.

I2. $37,3-37,6$. Die Haut ist recht weit gereizt.

I3. $36,3-37,0$.

I5. 36,7-37,0. Urticaria am ganzen Körper. Es hat sich eine Fistel an einer Stelle der Naht gebildet. Chin. omitte. Mage. sulf. und nat. sulf. aa $\mathrm{I}_{5}, \mathrm{O}$.

I6. 37,0-37,0. Hinten rechts sind noch in der Lunge Rasselgeräusche $\mathrm{zu}$ hören. Das Ekzem an der Wunde hat bedeutend abgenommen.

I7. 36,8-37,0. Urticaria verschwunden. Die Fistel scheidet fast gar nichts aus.

2I. Die Fistel hat sich geschlossen.

23. Patient wird in die Abteilung für Massage herüber geführt.

24. XI. Der Kranke verläßt in befriedigendem Zustande das Krankenhaus.

Den 20. I. Igog wurde der Kranke wegen einer Paralyse der rechten Hand zum zweiten Male in das Hospital aufgenommen. Jetzt war die Narbe am Halse hart und schmerzlos. Kein Pulsieren und kein Sausen. Auf der Resektionsstelle der Clavicula fühlt man schon einen sich neubildenden Knochen, die äußere Konfiguration dieser Gegend fast unverändert. Der Halsumfang ist $38 \mathrm{~cm}$. Auf der rechten Art. temporalis und der recht. a. radialis fehlt der Puls. Im Bereiche der rechten Seite des Gesichtes und des rechten Armes kein Ödem, die Hand ist leicht cyanotisch; der Kranke hat ein beständiges Kältegefühl in dieser 
Hand. Die Lähmung blieb ohne Veränderung wie vor der ersten Operation. II. Februar Operation.

Unter Chloroform- und o,oI Morphium-Narkose wird der Medialis des rechten Armes nebst seinen Ästen längs des Tractus der Schußwunde freigelegt. Der angedrungene und von Verwachsungen umgebene Nerv wird heil gefunden und aus den $8 \mathrm{~cm}$ langen Verwachsungen befreit.' Während der Operation ist kein arterielles Bluten vorhanden. Es bluten hauptsächlich die Hautgefäße. Hautnaht. Trockener Verband. Abends Temp. 36,o. Den I6. werden die Nähte entfernt. Prima intentio.

I8. Klagen über stechenden Schmerz in der rechten Hand und dem rechten Arm. Vorgeschrieben leichte Massage mit aktiver und passiver Bewegung.

23. Schmerzen im Arme bedeutend weniger.

28. Man merkt kleine aktive Bewegungen der Fingerextensoren. 6. III. Langsam aber zweifellos treten Fingerbewegungen ein.

Im eben beschriebenen Falle entstand das Aneurysma infolge einer Schußverletzung der Art. carotis communis und der Vena jugularis interna an der Stelle, wo die letztere in die Subclavia mündet. Es fing, wie es überhaupt in solchen Fällen vorkommt, mit einem diffusen pulsierenden Hämatome an, das sich später organisiert und verkleinert hat. Eine wallnußgroße langsam im Wuchse zunehmende Höhle hat sich mitten in dem fibrösen Bindegewebe der Schußwunde abgegrenzt und somit war auch das Aneurysma mit allen es umgebenden Geweben verwachsen. Das mußte sehr stark auf die Technik der Operation einwirken. Die Chirurgen wissen wie schwer es oft ist die Operation eines traumatischen arterio-venösen Aneurysmas zu Ende zu bringen. Hierbei fällt mir das von Herrn Prof. O p p e 1 operierte arterio venöse Aneurysma der Art. communis ein. Fast 4 Stunden hat sich der Chirurg bemüht, um die Verwachsungen freizulegen. 3 mal wurde die Vena jugul. interna mit nachfolgenden Einströmen von Luft traumatisiert. Es gelang nicht die Vene zu unterbinden. Man war genötigt, um sichere Blutstillung zu erzielen zwei Péan auf das peripherische Ende der Vene zu klemmen. Trotz der Unterbindung der Art. carotis communis gelang die Exstirpation des aneurysmatischen Sackes nicht. Man mußte sich mit einer partiellen Resektion des unteren Teiles des Aneurysma mit der des blutzuführenden Art. carotis und des blutabführenden V. jugularis begnügen. Der Kranke genas. 
Schon längst hat $\mathrm{Del}$ bet $\mathrm{festgestellt,} \mathrm{daB} \mathrm{die} \mathrm{einzige} \mathrm{radikale}$ operative Behandlung des arteriovenösen Aneurysmas in folgendem besteht: I. Unterbindung der Arterie und der Vene oberhalb und unterhalb des Aneurysma, 2. Offnen des Sackes mit nachfolgender Unterbindung aller in ihn sich mündenden Blutgefäße und 3. Exstirpieren des Sackes nach der Ligatur obengenannter Blutgefäße. Die Indikation zur Isolierung der Arterie von der Vene mit nachfolgender Nath dieser Gefäße ist so selten, daß man darüber nicht viel zu sprechen braucht. Von den gleichgenannten drei Methoden ist die letzte die radikalste. Es ist sehr interessant $\mathrm{zu}$ bemerken, daß nach $\mathrm{D}$ e $1 \mathrm{bet}$, die gewöhnliche Unterbindung der blutzuströmenden Arterie bei solchen Aneurysmen 4 mal mehr zur Gangrän führt als die drei obengenannten radikalen Methoden. Diese anfangs unbegreifbare Tatsache findet ihre Erklärung in der blutaufsaugenden Kraft der Vene wie es Prof. O p p e 1 bei seiner Operation eines subclavicularen arteriovenösen Aneurysma bewiesen hat. Das Exstirpieren des Aneurysma ist, wie gesagt, nicht immer möglich. Meistenteils treten unüberwindliche Schwierigkeiten auf. So hatte Pluyette und Bruneau im Jahre I905 7 neue Fälle von Unterbindungen des subclavicularen arteriovenösen Aneurysma gesammelt, in keinem dieser Fälle gelang es den Sack zu exstirpieren. Und in unserem Falle, bei der Beobachtung aller obenangeführten Regeln der operativen Behandlung waren wir genötigt, um den Sack zu exstirpieren, die Arterie wie auch die Vene oberhalb und unterhalb des Sackes zu unterbinden. Da aber das Aneurysma sich sehr niedrig in der Fossa juguluris befand, so war man genötigt, nicht die Art. communis sondern den Truncus anonymus zu unterbinden. Die Technik der Unterbindung des Truncus anonymus ist bis jetzt noch nicht völlig festgestellt. Verschiedene Chirurgen haben nur die leitenden Hauptprinzipien dieser Operation vorgeschlagen. Und es konnte auch nicht anders sein, da die Anonyma keine bestimmte Größe hat. Die Stelle ihrer Teilung in die Art. carotis communis und Art. subclavia befindet sich in normalen Fällen hinter der Articulatio sternoclavicularis, oft aber tiefer im Mediastinum, seltener über der Artericulatio im Bereiche des Halses.

Man kann die Methoden der Freilegung der Anonyma in ein- 
fache und komplizierte teilen. In der ersten Kategorie interessiert uns vor allen Dingen der Schnitt der Weichteile. Hier findèn wir die Pirogoffsche Methode, der nur einen Schnitt längs der Medianlinie des Halses und des Sternum führt. Zwischen dem Mm. sternohyoidei dringt er bis zu der Trachea vor, dann sucht er seitwärts von der Trachea die rechte Art. carotis communis und geht auf stumpfem Wege der Arterie entlang bis zur Anonyma.

Nach M ot $t$ wird ein winkelförmiger Schnitt längs dem inneren Rande der Mm. ster. cleid. mast. und dem oberen Rande der Clavicula gemacht, dann wird der somit erhaltene Hautlappen samt dem durchschnittenen St. cl. mast. nach oben und lateralwärts zurückgeschlagen. M o t $t$ durchschneidet auch den unteren Ansatz der Mm. sternohyoid. und sterno-thyreoid.; dann legt er die Art. carot. com. frei und geht wie in der obenbemerkten Weise auf die Anonyma über. Man kann aber diese zwei Methoden ungeachtet auf die bedeutende Erweiterung des Operationsfeldes im zweiten Falle nicht ganz befriedigend für das freie Operieren anerkennen. Sie sind leider nur für die Fälle der hohen Teilung der Anonyma und bei normalen Verhältnissen der Gewebe gut, was man aber nicht voraussehen kann. Auch kommen diese günstigen Verhältnisse nur bei Unterbindungen der Blutgefäße weit von dem traumatischen Aneurysma vor, zum Beispiel hauptsächlich beim Unterbinden der Anonyma infolge eines Aneurysma im dritten Abschnitte der Art. subclavia. Die Sache wird wesentlich schwieriger bei Veränderungen der Blutgefäße und des umgebenden Gewebes.

Es ist unbedingt nötig, um sicherer in dem Mediastin. anticum zu operieren, einen Teil des ihn bedeckenden Knochens zu entfernen. Unter den komplizierten Methoden der zweiten Kategorie mit den Manipulationen am Knochen sind die von B a r d e nhe uer und Milton nicht der Rede wert, da sie sehr kompliziert sind und auch ein sehr großes Trauma darbieten. Im Gegenteile verdient die Methode $\mathrm{Cur}$ t is völligster Achtung. $\mathrm{Zu}$ dem medianen Schnitte nach $\mathrm{P}$ i r o g of $\mathrm{f}$ bringt er noch einen queren in der Höhe des I. oder 2. Spat. intercostalis hinzu. In der Fossa jugularis wird ein Querschnitt der Fascie und des Periost des oberen Randes Manubrii sterni gemacht, von dessen hinteren Seite das Periost abgetrennt wird. Das Manubrium sterni wird 
in der medianen Linie und in der Höhe des horizontalen Hautschnittes durchmeißelt. Jetzt können die beiden Hälften des Manubrium sterni stark dilatiert werden, wozu man auch greift, um das hintere Periost zu durchschneiden. Nun vergrößert sich der Zwischenraum der beiden Hälften des Manubrium bis auf $2 \mathrm{~cm}$, was die Möglichkeit gibt, in der Tiefe des Mediastini antici zu manipulieren.

Diese Methode wurde sorgfältig von $\mathrm{W}$ wedensky an Leichen und Hunden bearbeitet. Ich zweifle aber ob man bei allen Leuten ein gleiches Auseinanderweichen des durchtrennten Manubrium erreichen kann, da man doch oft Verkalkungen der Rippenknorpel oder eine starke Entwickelung des Bandapparates usw. vorfindet. Diese Erschwerungen werden mit der temporären Resektion des ganzen Manubrium sterni - Methode R u e d i g e $\mathrm{r}$ R y d y g i e r - überwunden. Ein bogenförmiger Schnitt wird vom inneren Rande des rechten M. stern. cl. mast. links nach unten über die linke Articulatio sterno-clavicularis bis zum ersten Spatium intercostale dann quer zu dem entgegengesetzten Spatium intercostale der anderen Seite und zuletzt nach oben bis zum äußeren Rande des M. st. cl. mast. geführt, wo er auch endet. Nach der Discision der weichen Teile wird die linke Articulatio sterno clavicularis völlig geöffnet, der Knorpel der r. linken Rippe vom Brustbein getrennt, rechts nur discidiert. Das Brustbein und das rechte Schlüsselbein werden über einem mit Vorsicht unterlegtem Elevatorium durchsägt. Dieser ganze Haut-Fleisch-Knochenlappen wird nach oben geklappt und nach dem Operieren im Mediastinum wieder zurückgestellt. Die Verwertung dieser komplizierten, nur noch an Leichen vorgenommenen Methode gehört der Zukunft, doch kann man darin einen bedeutenden Fortschritt sehen.

M o ynihan unterband im Jahre I898 die Anonyma; erst exstirpierte er ein Aneurysma des dritten Abschnittes der Art. subclavia. Später bildete sich zentralwärts ein neues Aneurysma, die nach einem infolge eines Erysipels entstandenem Abszesse platzte und starke Blutung hervorrief. Die Operation bestand in Bildung eines Lappens, dessen Basis auf dem Halse ruhte. Der innere Teil der Clavicula und ein Teil des Manubrii sterni über der ersten rechten Rippe bis zur medianen Linie wurden reseziert. Der Hautlappen mit dem resezierten Knochen und dem M. st. 
cleid. mast. nach oben geklappt und nach Unterbindung der Art. carot. communis und Anonyma wieder an der früheren Stelle befestigt. Der Kranke starb eine Stunde nach der Operation (zitiert nach $\mathrm{J}$ a cobsth a l).

In meinem Falle erwartete ich große Schwierigkeiten, hauptsächlich starkes Bluten vieler venösen Collateralien, so daß ich nicht wagte eine zu sehr komplizierte Methode vorzunehmen, und resezierte nach $\mathrm{Ch}$ as a ig n a einen Teil des Manubrium sterni in Form eines zum Jugulum sich öffnenden Winkel, nachdem ich den Knochen vom Periost und dem weichen Gewebe freigelegt hatte. Er tat es mit einer Kettensäge, die er durch ein gebohrtes Loch führte, ich machte es mit der Luersschen Knochenschere - der Unterschied scheint unbedeutend zu sein. Außerdem resezierte ich das innere Drittel der rechten Clavicula streng subperiostal. Das Operationsfeld war sehr groß und man konnte frei im Mediast. antic. operieren. W w e d e nsky meint, daß der M. sterno cleid. mast. nach dem Durchtrennen an der Ansatzstelle sich stark kontrahiert und somit unangenehme Folgen nach sich zieht. Außerdem kann man befürchten, daß bedeutende Deformationen des Halses und funktionelle Störungen des M. st. cl. mast. und der rechten Wand könnten als Folge der Resektion des Manubrii sterni und der Clavicula hervorgerufen werden. Mein Fall ist ein Beweis der Grundlosigkeit dieser Befürchtungen: Fast gar keine Deformation, der Kranke kann unbehindert und schmerzlos alle Bewegungen im Schultergelenke machen.

Was die Ligatur selbst anbetrifft so scheint es mir, wie auch vielen anderen Autoren, nicht von Wichtigkeit zu sein, ob sie aus Katgut oder Seide besteht, obwohl ich bis jetzt beim Unterbinden größerer Arterien die Seide dem Katgut vorgezogen habe. Ein anderes Detail dieser Unterbindung interessiert schon lange die Chirurgen - soll man den das Blutgefä $B$ umschlingenden Faden stark zuziehen, oder nur so daß er den Blutstrom hemme. Doch scheint auch diese Frage nicht so wichtig zu sein, da in beiden Fällen die Intima des Blutgefässes dank der Kompression und Ernährungsstörung beschädigt wird. In meinem Falle war die Arterie stark unterbunden und die Festigkeit der thrombosierten Narbe war durch die Unschädlichkeit einer starken Excitation am Anfange der zweiten Narkose bewiesen. Die Frage ob 
überhaupt das Unterbinden der Anonyma zulässig sei, ist den meisten Chirurgen noch nicht klar. Das erklärt die Tatsache, daß davon sogar in den großen Handbüchern der Chirurgie nichts erwähnt wird. Nach der Unterbindung der Anonyma kann man Komplikationen I. vom Gehirne und dem rechten Auge, 2. von der rechten Hand erwarten. Nach der Statistik B u r n s starben nur 6 (I2 Proz.) am Chok, Anämie des Gehirnes und einmal an Erscheinungen der Hemiplegie (Min Ballance Lancet Igo2, Nr. I, nach J a c o bs th a 1 war das auch im Falle P i r o g o f f s).

Es ist nicht unnütz sich an die Statistik $\mathrm{P}$ oive $t$ (zit. bei $\mathrm{J}$ a c o bsth a l) zu erinnern, wo auf 63 Fälle einer Unterbindung der rechten Art. carot. com. allein oder mit der Art. subclavia infolge eines Aneurysma der Anonyma 6 Todesfälle sich einstellten wegen Komplikationen am Gehirne. In der Statistik des Herrn $\mathrm{J}$ a c o bsthal, die eine Fortsetzung derjenigen Dr. P oivet darstellt, sind auf 24 solcher Fälle 4. mit tödlichem Ausgange zu registrieren, verursacht durch Komplikationen am Gehirn. Andererseits finden wir nur einen Todesfall - nach einer Hemiplegie - unter 29 Fällen von Unterbindungen der rechten Art. carot. com. oder der Anonyma zum Teil mit einer Ligatur der rechten Art. subclavia und Art. vertebralis. Von ihnen sind 26 Fälle in der Statistik Souchons angeführt, die übrigen 3 Fälle sind von Jacobsthal, der sich an Souchons Statistik hält, beschrieben worden. Was die seltene Komplikation von seiten des Auges anbetrifft, so bestehen sie in einer Sehstörung, die sich bis zu einer vollen Blindheit steigern kann. Die Pupille bleibt eng, zuweilen kommt eine Vereiterung des Auges vor (D e $1 \mathrm{~b}$ e $t$ ). Die Komplikationen am Auge und Gehirne finden ihre Erklärung zum Teil in der Entstehung einer emporsteigenden Thrombose der Art. carotis comm. bis zu ihrer Verzweigung ( $\mathrm{L}$ e F ort, Gu i $\mathrm{n}$ a $\mathrm{rd}$ ), teils in der Bildung von Embolien aus den Gerinnseln der Art. carot. comm., die infolge des zurückströmenden Blutes aus der Carotis externa in die Carotis interna getrieben werden (D e l b e t). Diese Komplikationen sind laut Statistik selten und entstehen entweder beim Vorhandensein eines sehr langsamen retrograden Blutstromes in der Carot. externa, oder bei einer obwohl schwachen Infektion der Ligatur infolgedessen auch des Thrombus (D e 1 bet). Gu in a $\mathrm{r} d$ behauptet kategorisch, daß bei der 
Unterbindung der Art. carot. comm. die Komplikationen des Gehirnes nie eintreten, wenn die Anastomosen zwischen beiden Art. carot. ext. nicht unterbrochen sind (gewiß ist die Infektion ausgeschlossen); seine Begründungen basieren auf seine I5 Fälle von Unterbindungen der rechten Carotis communis und der rechten Art. subclavia bei Aneurysmen großer Gefäßen an der Basis. Jedenfalls führt das Unterbinden der Art. carot. communis nicht zu einer langdauernden Anämie des Gehirnes, da der collaterale Blutlauf stark entwickelt ist. Außèr den Anastomosen zwischen den Art. carot. exter., von denen soeben die Rede war, d. h. den Blutgefäßen der Lippen, des Gesichtes, des Schädels, der Kiefern, der Nase und der Zunge, gibt es noch weitere Anastomosen zwischen den Blutzirkulationssystemen beider Teile des Gehirnes - Circulus art. Willisii und Art. basilaris. Bei meinem Kranken traten Komplikationen des Gehirnes weder während noch nach der Operation auf. Die Schmerzen und das Ödem der rechten Hälfte des Gesichtes verschwanden und die völlige Genesung trat allmählich ein.

Was die gefährlichen Nacherscheinungen an der Hand betrifft, so kann man nur dasselbe von der Art. subclavia sagen, was von der Art. carotis comm. gesagt worden ist: Je höher die Arterie unterbunden wird, desto weniger Gefahr unterliegt die Hand bei ungestörten Collateralien. Das Unterbinden der Anonyma oder des I. Abschnittes der Art. subclavia infolge der Excision eines Aneurysma in der Fossa supraclavicularis oder Axillaris kann freilich eine Gangrän der Wand hervorrufen, aber bei dem normalen Verhalten, wie in meinem Falle, sichert der collaterale Blutkreis die Unversehrtheit der Hand. Es muß erwähnt werden, daB das Blut beim Unterbinden der Art anonyma und Art. carotis comm. in die Art. subclavia und axillaris durch folgende Wege eintritt: I. Art. vertebr. sin., Art. vertebr. dexta, subclavia, 2. Art. epigast. a. mammaria dex. subclavia, 3. A. thyreoidea inf. sin. a. thyreoid. dex. subclavia, 4. Aa. intercostales, Aa. subscapularis (thoracodorsales) und thoracica longa, Art. axillaris. AuBerdem haben eine große Bedeutung die in dem Unterhautzellgewebe verlaufenden Blutgefäße. Somit ist aus dem Gesagten klar, daß das Unterbinden der Anonyma bei vorhandenen Indikationen eine ebenso berechtigte Operation ist, wie das Unterbinden einer 
beliebigen großen Arterie. Zwar ist die Mortalität in der allgemeinen Statistik sehr groß - 77 Proz. Aber diese hohe Zahl hängt davon $a b$, daß in dieser Statistik Fälle der vorantiseptischen Zeit eingeschlossen sind, wo die Operierten an Sepsis oder an nachfolgender Blutung starben. Nehmen wir zur richtigen Schätzung dieser Operation Fälle der neueren Zeit, vom Jahre I8go bis zum heutigen Tage, so finden wir II Fälle einer Unterbindung der Anonyma, von denen 5 einen letalen Ausgang hatten und 6 zur Genesung kamen. Wenn wir aber die 5 vom Jahre Igoo beschriebenen Fälle ( $H$ er n a n d e $z, D$ e L a u p, B a 11 a n c e, Sh e e n, Burns) und auch meinen dazu nehmen, so haben wir 2 mit letalen Ausgange und 4 mit Genesung.

\section{Literaturverzeichnis.}

I. De 1 be t., P. Traite de Chirurgie Le Dent. et Delbet T. 4 .

2. Burns, W. The Journ of the Amer. Med. Assoc. I908, Nov.

3. Guin ard, A. Revue de Chirurgia 1909 , Nr. 2.

4. Ja cobstha1, H. Deutsche Zeitschr. f. Chir. Ig02, S. 551.

5. Derselbe. Deutsche Zeitschr. f. Chir. I903, S. 239.

6. Souchon, E. Agn. of surn 1895 .

7. Shee 11, W. Ann. of surg. 1905; Brit. med. Journ. I900.

8. Ruediger Rydygier, L. Wiener klin. Wochenschr. I906, Nr. 50.

9. Plyctte et Brunea u. Revue de Chirurgic I905, T. 2.

ı. W w e d e n sky, A. Russkij chirurg, Archiv I906, H. I.

II. v. Op pel, W. Russkij chirurg. Archiv 1905, H. 5. 